PROFESIONALES Y HERRAMIENTAS PARA EL DESARROLLO LOCAL Y SUS SINERGIAS TERRITORIALES. EVALUACIÓN Y PROPUESTAS DE FUTURO IX Coloquio Nacional de Desarrollo Local del GTDL-AGE 

ANTONIO MARTÍNEZ PUCHE, XAVIER AMAT MONTESINOS, ISABEL SANCHO CARBONELL y DANIEL SANCHIZ CASTAÑO (EDS.)

\section{PROFESIONALES Y HERRAMIENTAS PARA EL DESARROLLO LOCAL Y SUS SINERGIAS TERRITORIALES. EVALUACIÓN Y PROPUESTAS DE FUTURO}

IX Coloquio Nacional de Desarrollo Local del GTDL-AGE

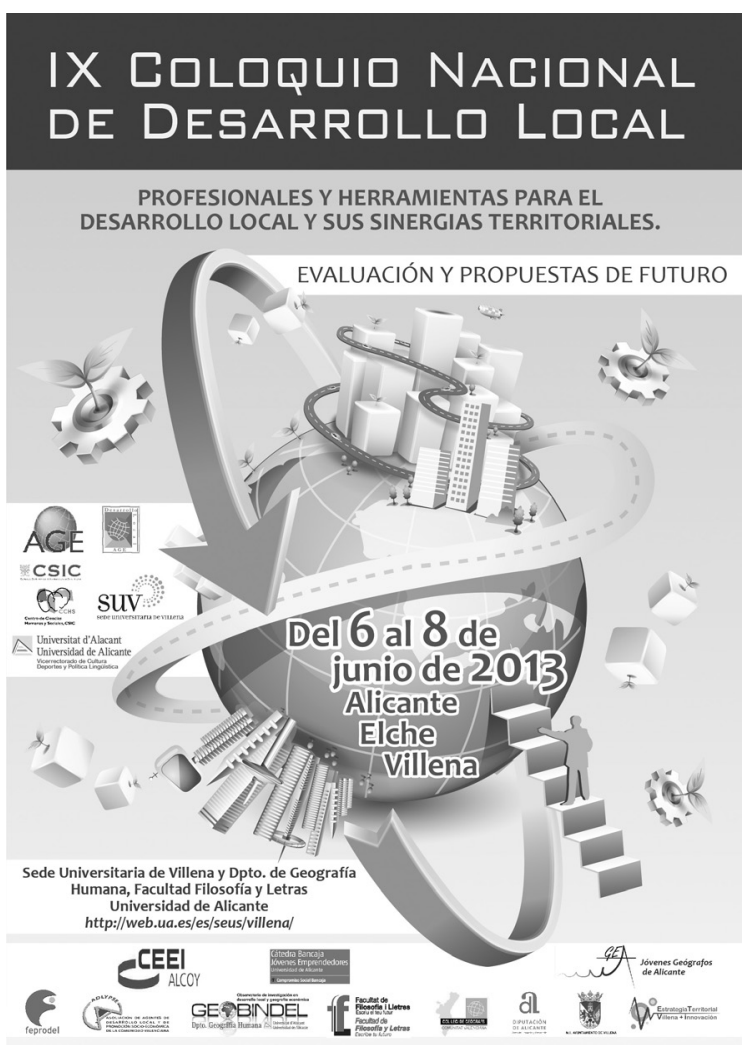


Este libro ha sido examinado y valorado por evaluadores ajenos a la Universidad de Alicante, con el fin de garantizar la calidad científica del mismo.

Publicacions de la Universitat d'Alacant

03690 Sant Vicent del Raspeig

Publicaciones@ua.es

http://publicaciones.ua.es

Telèfon: 965903480

(C) Antonio Martínez Puche, Xavier Amat Montesinos,

Isabel Sancho Carbonell y Daniel Sanchiz Castaño (eds.), 2016

(C) d'aquesta edició: Universitat d'Alacant

ISBN: 978-84-16724-00-0

Dipòsit legal: A 92-2016

Disseny de coberta: candela ink

Composició: Página Maestra (Miguel Ángel Sánchez Hernández)

Impressió i enquadernació: Guada Impresores

\section{unte \\ Unión de Editoriales
Universitarias Españolas \\ WWW.une.es
WWA}

Esta editorial es miembro de la UNE, cosa que garantiza la difusión y comercialización nacional y internacional de sus publicaciones.

Reservados todos los derechos. Cualquier forma de reproducción, distribución, comunicación pública o transformación de esta obra sólo puede ser realizada con la autorización de sus titulares, salvo excepción prevista por la ley. Diríjase a CEDRO (Centro Español de Derechos Repográficos, www.cedro.org) si necesita fotocopias o escanear algún fragmento de esta obra. 


\title{
POSIBILIDADES DEL DESARROLLO LOCAL COMO SISTEMA DE ENCADENAMIENTOS PRODUCTIVOS EN LA REGIÓN SUR DEL ESTADO DE TLAXCALA, MÉXICO
}

\author{
María del Pilar Jiménez Márquez \\ Universidad del Caribe \\ mjimenez@ucaribe.edu.mx
}

\section{RESUMEN}

El objetivo del presente trabajo es mostrar algunos resultados importantes del estudio sobre el desarrollo local analizado desde la perspectiva de los sistemas de encadenamientos de las unidades económicas de los sectores productivos, factores socioculturales y representantes de los gobiernos locales de los municipios de la región sur del estado de Tlaxcala, México. Investigación que destaca la necesidad de políticas descentralizadas y la importancia de los elementos endógenos locales.

Palabras clave: Desarrollo local, encadenamientos productivos y tejido productivo.

LOCAL DEVELOPMENT POSSIBILITIES AS A SYSTEM OF PRODUCTION LIAISONS IN THE SOUTHERN REGION OF THE STATE OF TLAXCALA, MEXICO

\begin{abstract}
The objective of this paper is in order to offer an overview about some of the most important results from the study about local development, by analyzing from the production chain system perspective of the economic unit from different productive sectors, socio-cultural factors and municipal government local representatives from the southern region of the city of Tlaxcala, Mexico. This paper is a research that outlines the necessity of decentralized policies and the importance of local endogen elements.
\end{abstract}


Key words: Local development, production chain systems and productive networks.

\section{INTRODUCCIÓN}

A fin de potenciar estructuralmente los recursos que construyen el territorio, es importante promover entre los actores del medio ambiente estrategias integrales en las regiones. Así lo destacan estudiosos del desarrollo local, para quienes, además, es indispensable conocer los elementos que conforman un territorio, la forma como se relacionan dichos elementos, así como identificar los canales que deben fortalecerse, con el propósito de exponer las propuestas idóneas. En tal sentido, el conocimiento del tejido productivo local es trascendental. No solo porque da origen a la actividad económica local, sino también porque se vincula con prácticas socioculturales, valores, tradiciones y prácticas cotidianas que realizan los agentes productivos locales en el territorio.

Asimismo, es importante considerar a los agentes públicos, encargados de instrumentar los programas político-administrativos. Estos programas deben estar encaminados a aplicarse a la población local atendiendo a las condiciones sociales, culturales y de recursos naturales con que cuenta la comunidad. Deben aplicarse de tal forma que en la interacción de los agentes locales (productivos, socioculturales y públicos) se generen sinergias que, en conjunto, logren fortalecer las relaciones entre los actores locales, y propicien encadenamientos que se conviertan, en un primer momento, en ancla del desarrollo local en el territorio para después encadenarse con otras actividades productivas hacia fuera, que a su vez se transformen en detonadoras de su propio desarrollo.

Sin embargo, el primer reto que enfrenta el desarrollo local para emplearse como estrategia de desarrollo es la falta de conocimiento de las formas de relaciones que se generan entre los agentes productivos, socioculturales y político administrativos, en países latinoamericanos y de forma particular en México, que es el caso al que haremos referencia. Los entornos locales han quedado inmersos en las dinámicas nacionales y globales debido a la ejecución de políticas gubernamentales centralizadas que difícilmente han incluido el reconocimiento de las dinámicas de menor escala. En consecuencia, no hay un nivel de información local y existe escasa posibilidad de articular los factores productivos, socioculturales y político-administrativos de los territorios locales para facilitarles desarrollar sus potencialidades internas. En tal sentido, pareciera una necesidad destacar mediante resultados de estudios, la importancia que tiene potenciar los elementos o recursos locales y mostrar los resultados importantes que dejan esas pequeñas pistas de articulación que los agentes productivos de pequeñas escalas logran crear, a pesar de no ser prioridad en la ejecución de políticas dirigidas a favorecerlos y promover su fortalecimiento. 
En tal sentido, el presente trabajo tiene la intención de presentar el análisis de las relaciones que se generan al interior de la región sur del estado de Tlaxcala, México, lugar en el que encontramos diferencias y concentraciones en el nivel de las actividades productivas, y es frecuente la combinación de áreas rurales y urbanas. Esta región se caracteriza por quedar incluida en programas de carácter intermunicipal, interestatal, nacional e internacional.

2. El desarkollo local como sistema de encadenamientos productivos. PreCISIONES TEÓRICAS

Los desafíos epistémicos se encuentran en buscar la construcción teórica de los procesos y relaciones que se construyen en las regiones en el campo de lo local, de lo micro (MONCAYO, 2001:91-116). El fin del conocimiento es la búsqueda de la compresión de la realidad como una realidad construida. Es importante anotar que no es la dinámica social que nos lleve a cualquier dirección, las alternativas son resultado de los límites creados por las acciones presentes de los propios sujetos sociales donde se eligen los nuevos caminos. Los niveles de análisis, estudio y reflexión exigen que se de la identificación de los actores locales, su reconocimiento y respeto en los distintos campos epistémicos, se requiere del estudio de las relaciones que se originan entre los actores locales en sus distintas dimensiones, identificando que el estudio de los agregados micro da lo macro (MEJÍA, 2008: 1-13).

El fin del conocimiento social es hacer comprensible la acción como posibilidad y no el establecimiento de la acción según regularidades inalterables y deterministas. La sociedad es un sistema muy complejo, es una dialéctica de interrelaciones entre los individuos con la totalidad social y la totalidad social con los individuos, y a medida que pasa el tiempo, la constante es la emergencia de nuevas realidades (LUHMANN (1973) en MEJÍA 2008: 1-13). La realidad es un mundo en proceso de construcción, donde los actores sociales con sus múltiples combinaciones aleatorias, sus incertidumbres, conductas contradictorias tienen un papel activo en el desarrollo del mundo real. El fin del conocimiento es la búsqueda de la comprensión de la realidad como una realidad construida. Es importante anotar que no es la dinámica social que nos lleve a cualquier dirección, las alternativas son resultado de los límites creados por las acciones presentes de los propios sujetos sociales donde se eligen los nuevos caminos, estudiar la sociedad es el reconocimiento de sus posibilidades creativas (MEJÍA, 2008: 1-13). La sobreabundancia de relaciones, de posibilidades, de conexiones que rebasa las relaciones biunívoca y lineal de elemento con elemento reconociendo la complejidad y precisando que no se trata de un sistema cerrado si no que existe en un entorno, lo que le permite caracterizarlo haciéndolo autopoiético y autorreferente; el primero porque es un sistema que crea su propia estructura y los elementos de que se compone, el segundo porque 
incluye en su misma constitución la diferencia con respecto a su entorno. Así que el sistema autorreferente incluye un gran dinamismo que se sustenta en la observación y la diferencia. La observación es una actividad fundamental para los sistemas autorreferentes mediante la cual se observan a sí mismos y observan cuanto se encuentran en su entorno, consiguiendo, establecer determinados procedimientos de selección y reducir la complejidad del entorno que les rodea. La observación es siempre una operación y tiene un radical carácter dinámico, se encuentra íntimamente ligado con el concepto de diferencia que ocupa un lugar central y donde la diferencia queda privilegiada sobre todo concepto de unidad. Todo aquello que pueda ser considerado unidad es, una unidad de diferencias por lo que es una unidad múltiple (LUHMANN, 1990: 16).

En ese dinamismo y diferencia, el conjunto de relaciones que existe entre los elementos de un sistema (LUHMANN, 1990: 16), la operación distinta que tiene cada subsistema y la necesidad de un tratamiento independiente, en tal sentido, (MONCAYO, 2001:91-116) propone la necesidad de un abordaje teórico desde lo local y desde el reconocimiento de una dialéctica de interrelaciones entre los individuos con la totalidad social y la totalidad social con los individuos. Proponiendo un abordaje del conocimiento desde un enfoque constructivista, reconociendo la emergencia de las nuevas realidades y la necesidad de distintas explicaciones. Bajo el reconocimiento de la complejidad y de la multiplicidad de relaciones entre los elementos que caracterizan a un sistema es que nos proponemos analizar las relaciones que se generan en el territorio revisado bajo los conceptos del desarrollo local que surge como proposición que desafía a la propuesta globalizadora (MEJÍA, 2008:1-13), pero también a la perspectiva unificadora o eurocentrista (WALLERSTEIN, 2002:157-296) y que a través de esta modesta investigación se hace un abordaje acercándose a lo que los principales conceptos del desarrollo local proponen que es fortalecer en las regiones las sinergias necesarias entre los actores del medio ambiente para potenciar de forma estructural los recursos que construyen el territorio que es uno de los principales postulados del desarrollo local. Para tal fin, es indispensable conocer los actores que conforman un territorio, la forma como se relacionan, así como identificar las relaciones que han construido y las que se van generando en los pequeños espacios. Que también es importante revisar bajo las características de cada subsistema.

\subsection{El desarrollo local}

El desarrollo local ha sido definido como un conjunto de relaciones y eslabonamientos de los recursos productivos y comerciales relevantes que dan paso a la eficiencia productiva, a la competitividad de la base económica de un determinado territorio y al aprovechamiento de las oportunidades del dinamismo exógeno. Para el autor, el desarrollo local es un enfoque territorial de "abajo 
hacia arriba", que busca la intervención de los tres niveles de gobierno e incluye, en un enfoque integrado, los aspectos de carácter ambiental, cultural, social, institucional y de desarrollo humano desde un punto de vista del territorio (ALBURQUERQUE, 2004:2).

Así, este autor aporta un enfoque integrado que incluye la intervención de los tres niveles de gobierno en un proceso de abajo hacia arriba, así como relaciones y eslabonamientos de los recursos productivos y comerciales en pro de la eficiencia productiva, para aprovechar el dinamismo exógeno.

El desarrollo local también es abordado como algo diferente al orden mundial impuesto que atribuye funciones especializadas a las diversas regiones del mundo. Por tanto, la diferencia es aquella generada en el espesor de cada proceso histórico. Ligado a la constitución de cada identidad colectiva, es necesario tener en cuenta, por tanto, la diversidad de los procesos locales. Las referencias identitarias fuertes, aquellas que actúan sobre el potencial socioeconómico aumentando su capacidad de respuesta, se encuentran en un territorio bien preciso, cuyos límites son conocidos por los miembros del grupo. Las sociedades locales existen en territorios cargados de huellas del pasado. El espacio no es neutro; expresa la historia de los hombres, sus conflictos y sus sistemas de vida, sus trabajos y creencias. La memoria colectiva da un sentido a la relación entre pasado, presente y proyecto, expresando así los contenidos profundos de la identidad colectiva. El retorno al pasado a través de la memoria, la lectura de las huellas que permiten reconocerse en una historia, es una condición de la acción (AROCENA, 1995: 10-55).

Por tanto, (AROCENA, 1995: 10-55) plantea el desarrollo local desde el territorio también y lo precisa como la diferencia que da el "grueso de cada proceso histórico", unido a la identidad colectiva, destacando la memoria colectiva, el sistema de vida y la historia de los hombres que actúan sobre el potencial económico.

El territorio tiene un conjunto de recursos materiales, humanos, institucionales y culturales que constituyen su potencial de desarrollo. Éste se expresa a través de la estructura productiva, el mercado de trabajo, la capacidad empresarial y el conocimiento tecnológico, las infraestructuras de soporte y acogida, el sistema institucional y político y su patrimonio histórico cultural. El autor destaca que sobre estas bases cada economía articula sus procesos de crecimiento y cambio estructural y, como consecuencia de la interacción de las empresas y los demás actores económicos en los mercados, se obtienen resultados muy diferentes que dan lugar a una gran variedad de situaciones, proyectos y procesos económicos, sociales y políticos (VÁZQUEZ, 2005:143-154). Esto señala la importancia que tienen los espacios locales, los recursos con que cuenta y la forma particular de cómo se relacionan.

La visión se amplía cuando se abordan el desarrollo y la dinámica de los sistemas productivos desde una óptica territorial. Ellos dan una gran impor- 
tancia a las iniciativas de los actores locales, a través de sus decisiones de inversión y de la participación en la formulación y ejecución de las políticas. Señalan que el progreso económico de un territorio solo es posible cuando las empresas y los demás actores del territorio interactúan entre sí, se organizan y realizan sus inversiones dirigidas a desarrollar la economía y la sociedad local. La identidad territorial permite potenciar la competitividad de las empresas y de la economía local estimulando los activos intangibles (marcas, derechos de propiedad, denominación de origen y calidad organizativa). Esto dará como resultado contar con un tejido productivo fortalecido a partir del sistema territorial de innovación, así como la capacidad de un territorio para ahorrar e invertir los beneficios generados por su actividad productiva en el propio territorio y promover el desarrollo diversificado de la economía, además de adoptar su propia estrategia de desarrollo, lo que está asociado con procesos de descentralización (JOHN FRIEDMAN y WALTER ROH (1979) en VÁZQUEZ, 2005: 143-154).

A partir del análisis conceptual del desarrollo local, se identifica que desde el territorio se determinan una serie de relaciones y eslabonamientos de recursos. Estas relaciones permiten entender el territorio como el espacio donde se desarrollan actividades socioeconómicas, políticas y culturales que propician herencias transgeneracionales vinculadas con la construcción social del día a día. Aquí confluyen los recursos del sistema productivo de los territorios pequeños. Este sistema está representado por las empresas-empresarios locales (unidades productivas) y por los recursos político-administrativos, lo cual se refleja en las políticas públicas o programas gubernamentales que inciden en el desarrollo local. En el desarrollo local, el territorio es el recurso inminentemente indispensable para su explicación.

De aquí se desprende un concepto más contenido en el desarrollo local relacionado con el sistema que se genera al interior del territorio, entendido desde la perspectiva de (LUHMANN, 2007: 77-126) como sistema dinámico; porque mantiene comunicación entre sus propios elementos, puede diferenciarse en distintos subsistemas, cada uno de ellos cerrado y autorreferente, que poseen un ámbito determinado de comunicaciones y de operación, que limitan su entorno y reducen la complejidad de un modo especializado. La sociedad se diferencia progresivamente, a lo largo de la evolución temporal y de la historia en diferentes subsistemas sociales. Una sociedad avanzada será siempre una sociedad altamente diferenciada con diferentes ámbitos de comunicación que son los diferentes subsistemas sociales. Con ello se ha logrado un elevado nivel de especialización en las diferentes funciones de la sociedad, que contribuye a reducir eficazmente la progresiva complejidad a la que la sociedad debe enfrentarse (IZUZQUIZA, 1990: 8-39).

En ese sentido, la interacción entre los recursos que forman la base productiva y los agentes locales forman parte de un sistema que les permite man- 
tenerse comunicados, así como crear sus propias estructuras por el intercambio de información, generando relaciones entre sí -autopoiesis (LUHMANN, 2007:118-126)- y marcando diferencias en su propio entorno. Esta situación es semejante a la que se presenta con los elementos o recursos que forman parte del sistema en el desarrollo local en un territorio, los cuales se encuentran cohesionados por una serie de relaciones de carácter político-administrativo, sociocultural y productivo, que les permite establecer mecanismos propios de comunicación con una sobreabundancia de relaciones, de posibilidades, de conexiones, de modo que la información que se intercambia en la comunicación no es biunívoca y lineal de elemento con elemento (LUHMANN, 1990:16).

Así también, es importante destacar lo siguiente: en un sistema integrado, los elementos o recursos (empresas-empresarios, programas públicos y características socio-culturales que intervienen en el proceso de producción) del desarrollo local se unen y hacen que todos ellos interactúen, estableciendo entre ellos una relación de comunicación e información. Esto da paso a la autorreferencia (LUHMANN, 1990:16) -expresada como la diferencia entre el sistema y su propio entorno-, pero que entre estos elementos existe una comunicación que permite el intercambio de información entre los elementos del sistema (IZUZQUIZA, 1990: 8-39). Movidas por representaciones relativas al intercambio de la materia o por representaciones del tipo input/ output,... por la apertura del sistema y su dependencia del medio ambiente, están en condiciones de reforzar su distinción con respecto al entorno (LUHMANN, 2007:77).

Por ello, los elementos o recursos del sistema de un territorio nos permiten conocer la funcionalidad que se da en el desarrollo local, e identificar el grado de integración de sus elementos y la dependencia que se genera entre ellos -en el sentido autopoiético que tienen las partes para ir generando su propia estructura y sus propios componentes (IZUZQUIZA, 1990: 8-39)-. Permite asimismo observar el proceso de autorreferencia que se da en el mismo sistema en el momento de los cambios a escala global que impactan en los espacios locales, como es el caso del dinamismo exógeno.

Un sistema (operación de comunicación) lleva a cabo este proceso (LUHMANN, 1990:16). Se efectúa mediante una diferencia en la medida en que una operación se enlaza, se traba con otra de su mismo tipo y va dejando fuera todo lo demás. Es decir, fuera del sistema, en el entorno, acontecen simultáneamente otras cosas. Estas otras cosas suceden en un mundo que solo tiene significado para el sistema en el momento en que pueda enlazar esos acontecimientos a la comunicación. El sistema, debido a que tiene que decidir si enlaza una comunicación con otra, necesariamente debe disponer de capacidad de observar, de percibir, lo que embona con él y lo que no embona. Un sistema, entonces, que puede controlar sus posibilidades de enlace, debe disponer de autoobservación; o dicho de otra manera: la comunicación posee capacidad de autoobservarse, 
sobre todo cuando ya está puesto en marcha un lenguaje para la comunicación y se tiene un repertorio de signos estandarizados (LUHMANN: 2007:90).

Lo que se estaría sugiriendo aquí es la posibilidad que tiene el desarrollo local de insertar las externalidades a su sistema para su reproducción. Esto representaría incluir elementos al sistema que fortalezcan los recursos ya presentes en el mismo (empresas-empresarios locales -unidades productivas-, políticas públicas o programas gubernamentales) que dan origen al desarrollo local. Con ello también se apoyaría la idea sugerida por (VÁZQUEZ, 2005:142) acerca de la visión bottom-up, que ha ido progresivamente fortaleciéndose en la formulación de las políticas de desarrollo de las ciudades y regiones. La cuestión clave es cómo conseguir que las economías locales estén más integradas en la economía internacional y lograr que sus sistemas productivos sean más competitivos, sin que ello signifique que dependan del exterior.

Para que la dependencia externa no ocurra es necesario fortalecer el funcionamiento y las relaciones entre los elementos del sistema al interior. Esto nos lleva a precisar los problemas de cada uno de los "recursos productivos", como los llama Polèse, para conocer sus límites y estar fuerte en las causas que impulsen su desarrollo. En su forma más avanzada, (VÁZQUEZ, 2005:143) asume que los recursos locales se organizan formando redes que le sirven para estimular el conocimiento y el aprendizaje sobre la dinámica del sistema productivo y de las instituciones, y para acordar iniciativas y ejecutar las acciones que integran la estrategia de desarrollo.

El desarrollo local como sistema integrado hace especial hincapié en la integración de todas las potencialidades de aprovechamiento de los recursos existentes en la zona. Intenta minimizar la utilización de tecnologías externas, poniendo especial énfasis en la movilización y el desarrollo de los recursos humanos y en la consecución de un acceso más equitativo a los medios de producción y a una más justa distribución de la renta. El desarrollo local como sistema abarca una política global que incluye aspectos de descentralización administrativa, organización de la población, ordenamiento del territorio y dotación de infraestructuras y servicios, etc. En ese sentido, se fortalecen las actividades económicas y socioculturales utilizando fundamentalmente sus propios recursos humanos y materiales. En esta parte del sistema, la iniciativa privada adquiere un papel preponderante. Por ello, se requieren nuevas fórmulas de colaboración empresarial y de participación social (PADILLAS et al, 2006: http://www.monografias.com/trabajos39/concepto-desarrollo-local/conceptodesarrollo-local2.shtml).

Por lo tanto, el sistema de encadenamientos en el desarrollo local sugiere la fortaleza de los recursos locales hacia dentro y el aprovechamiento de los recursos o elementos que el mismo sistema local requiera del exterior para robustecerse. Lo importante es "endogeneizar" las oportunidades externas (ALBURQUERQUE, 2004:2) generando los encadenamientos productivos 
que permitan el crecimiento hacia el interior y a partir de ello integrarse al exterior.

Los grandes retos que enfrenta el desarrollo local estriba, (ALBURQUERQUE, 1997: 313-325), en actitudes tradicionales que deben ser superadas en la gestión pública local, tales como el frecuente desconocimiento de las circunstancias, problemas y potencialidades del sistema productivo local y del tejido de empresas existente; la desconfianza entre agentes sociales, en especial entre autoridades y empresarios locales; la limitada vida asociativa y de interlocución social entre autoridades locales y vecinos; una visión de las funciones municipales limitada a la prestación de servicios sociales y urbanísticos; la desatención de las zonas rurales y la inhibición ante problemas importantes ante el riesgo del revés electoral.

\section{Región sur de Tlaxcala, México}

Los municipios de la región sur: Acuamanala de Miguel Hidalgo, Ixtacuixtla de Mariano Matamoros, Mazatecocho de José María Morelos, Natívitas, Papalotla de Xicoténcatl, San Jerónimo Zacualpan, San Juan Huactzinco, San Lorenzo Axocomanitla, San Pablo del Monte, Santa Apolonia Teacalco, Santa Catarina Ayometla, Santa Cruz Quilehtla, Tenancingo, Teolocholco, Tepetitla de Lardizábal, Tepeyanco, Tetlatlahuca, Xicohtzinco y Zacatelco (mapa 1), ocupan una extensión territorial de $578.89 \mathrm{~K}^{2}$, y ocupan el 14.28 por ciento del total del territorio del estado de Tlaxcala (cuadro 1). Se encuentran entre las latitudes norte $19^{\circ} 7^{\prime}$ y $19^{\circ} 20^{\prime}$ así como entre las longitudes oeste que van desde $98^{\circ}$ $11^{\prime}$ y $98^{\circ} 23^{\prime}$. De acuerdo con datos del censo de población del (INEGI, 2010: 15), la región del sur registró una población total de 331,456 habitantes, lo que representó $28.33 \%$ de la población del estado y, en consecuencia, una densidad de 559 habitantes por kilómetro cuadrado.

Cuadro 1. Extensión territorial de municipios del sur del estado de Tlaxcala

\begin{tabular}{|c|c|c|}
\hline \multirow{2}{*}{$\begin{array}{c}\text { Municipios de la región } \\
\text { sur del estado }\end{array}$} & Superficie en $\mathbf{K m}^{\mathbf{2}}$ & $\begin{array}{c}\text { Porcentaje con respecto } \\
\text { al territorio estatal }\end{array}$ \\
\cline { 2 - 3 } & 578.89 & 14.28 \\
\hline
\end{tabular}

Fuente: Elaboración propia con base en información del Instituto Nacional de Estadística, Geografía e Informática, Censo de Población y Vivienda, 2010, México, 2011. 


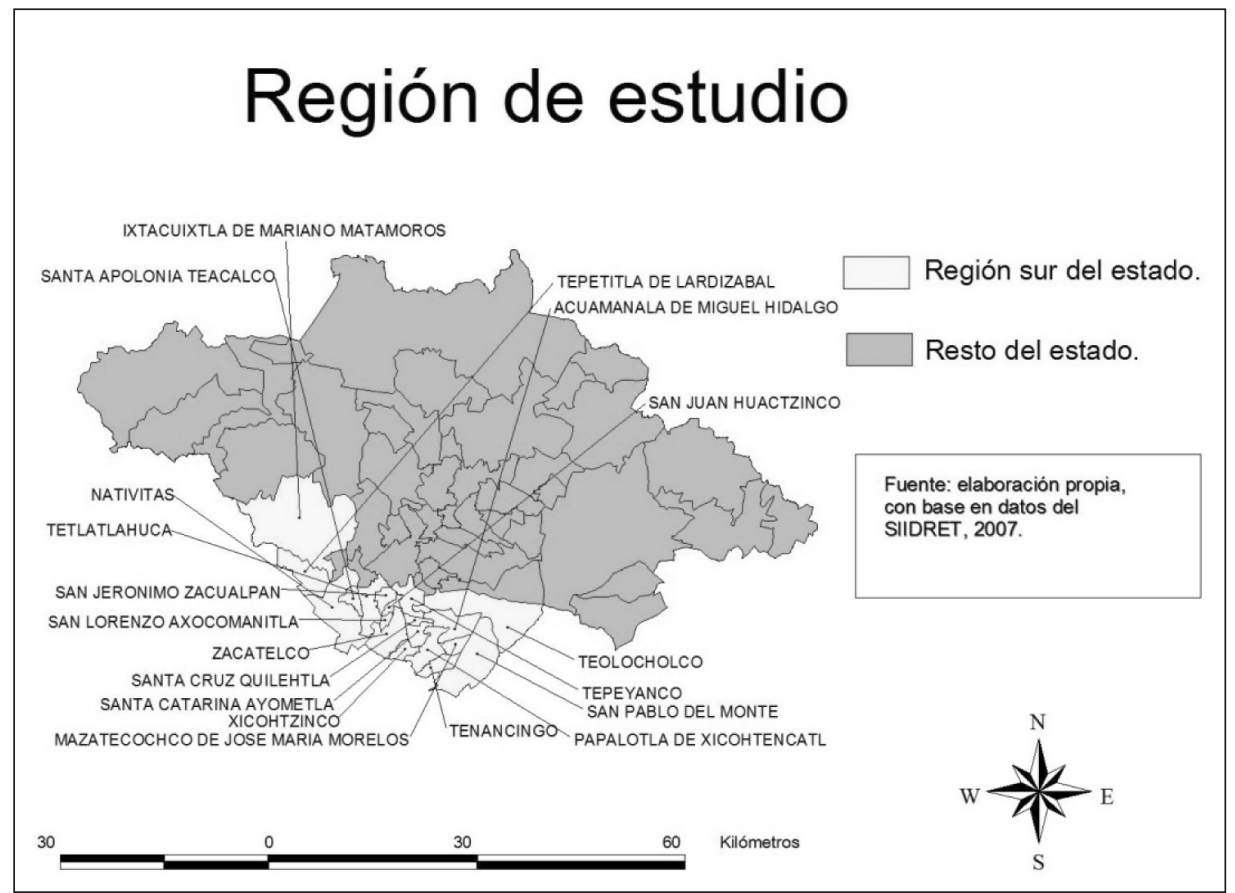

Mapa 1. Ámbito de estudio.

Fuente: elaboración propia.

La región sur del estado de Tlaxcala ha sido incluida en dos determinaciones nacionales de regionalización, la primera que buscaba la desconcentración de la industria en la Ciudad de México, capital del País, mediante la instrumentación de la política de polos de desarrollo, que originó cambios en el tejido productivo y en las formas de organización social en la región sur del estado de Tlaxcala, puesto en marcha en los años setentas, motivado por los gobiernos estatales que de acuerdo a (ROSALES, 2003: 131-163) promovían la industrialización como una solución al agudo problema social que vivía la entidad en ese momento, coincidiendo el tiempo que vivía Tlaxcala con las decisiones que tomaba el gobierno federal al emitir decretos de desconcentración industrial, así como la creación de parques y ciudades industriales, en julio de 1972, quedando Tlaxcala incluida en la zona III de Descentralización Industrial y Desarrollo Regional. Programa que fundamentalmente benefició a los municipios del centro y sur de la entidad que generaron mayores relaciones de dependencia con los estados vecinos del Estado de México, Ciudad de México y Puebla.

El proceso bajo el cual Tlaxcala enfrenta su segunda forma de ser considerada en los programas de regionalización, es a partir del programa instrumentado en la primera década del siglo XXI, denominado Programa de Desarrollo 
Región Centro País (PDRCP), que plantea un nivel de planeación integral a escala meso-regional, y en el contenido del programa propone la articulación de aspectos básicos de desarrollo económico regional, desarrollo social regional del medio ambiente y de ordenamiento territorial, que en estricto sentido los recursos destinados a la ejecución del PDRCP no han considerado los distintos ámbitos planteados en sus contenidos, pues la parte instrumentada está encaminada a la generación de infraestructura carretera dejando de lado el tejido productivo y pone especial interés en municipios de la región sur del estado que han sido considerados antes en otros programas de carácter internacional.

El grado de importancia que tiene la dinámica económica, política y social de los municipios del sur de Tlaxcala que forman parte de la zona conurbada Puebla-Tlaxcala, tiene implicaciones que rebasan los límites de los municipios del estado y se articulan en el sistema de la región centro país, así como de otros programas de carácter internacional.

La evidencia reflejada en las políticas que han promovido la inversión y el desarrollo en la entidad tlaxcalteca, muestran que el esfuerzo por promover el desarrollo regional ha dejado desprotegida las cuestiones locales de los municipios sobre los que se han caído directamente los recursos de los programas aplicados, originando desequilibrios y concentraciones de servicios, infraestructura y población como es el caso de los procesos que se registran en los municipios de estudio.

La forma en cómo se ha llevado a cabo la promoción del desarrollo mediante la aplicación de políticas y aplicaciones de programas de carácter vertical no han permitido identificar las fortalezas locales, limitando con ello al fortalecimiento del conocimiento y reconocimiento de las fortalezas de los elementos locales.

Por tanto, en el nivel de decisiones, planes y programas que se implementen en los municipios del sur del estado de Tlaxcala, se requiere ahora de máxima atención estratégica, así como de un conocimiento de lo que ocurre al interior de los mismos municipios. Es necesario conocer la forma como se relacionan los actores locales y evaluar las políticas aplicadas de modo que se puedan proponer cambios.

Para ello es necesario que los programas propuestos que buscan elevar la productividad de la región, consideren la participación de los actores locales, y propongan nuevas formas de microrregionalización que permitan desde lo local generar propuestas para disminuir las diferencias entre regiones. La dinámica actual obliga a dinamizar el proceso, pero las medidas que se requieren para elevar la productividad de las regiones de manera integral, debe fundamentarse en el conocimiento de lo local, en la consideración de los recursos, tanto humanos como de infraestructura, tanto naturales como financieros, y no solo basar la productividad en la creación de infraestructura carretera. 


\section{Relaciones PROdUctivas en la Región SUR del estado de TlaXCala}

La caracterización del tejido productivo de la región sur del estado de Tlaxcala presenta relaciones productivas y comerciales, se producen entre los mismos municipios que conforman la región sur del estado de Tlaxcala, entre la región sur del estado de Tlaxcala y otros estados, así como entre la región sur del estado de Tlaxcala y otros países, registrándose por tanto exportaciones que permiten la inserción al sector externo.

Las actividades industriales de las unidades económicas de la región sur del estado de Tlaxcala, que alcanzan un mercado a escala internacional, tienen un impacto importante en la región sur del estado de Tlaxcala, así como en el municipio en donde están localizadas. Las unidades económicas de la región sur del estado de Tlaxcala logran espacios para vender sus productos de manera más amplia, pues durante la investigación se identificó que su radio de influencia tiene escalas locales, regionales, nacionales e internacionales.

A diferencia de las actividades comerciales que alcanzan un mercado a escala regional, pues sus productos se venden en la región sur del estado de Tlaxcala, es un comercio principalmente dedicado a la venta al por menor y en niveles mínimos al por mayor.

Las unidades económicas dedicadas a las actividades de servicios logran extender su mercado a nivel local y regional. E identificamos que sus productos se venden de manera mínima en otros estados. Sin embargo, el alcance de los mercados que tienen no es lo más importante. Lo que es trascendental para capitalizar la "productividad" que plantea (ALBURQUERQUE, 2004:6-7), es la articulación que ya tienen entre las unidades económicas de las actividades secundarias, comerciales y de servicios. Si bien registramos, y se hace evidente, el nivel de intercambio que se mantiene con el municipio de San Martín Texmelucan, Pue., así como con el municipio de Puebla, que ocupan un lugar importante en el abastecimiento por ser mercados de carácter regional. Es aún más significativo que incluso con su cercanía, los productos locales mantengan un impacto local, regional y hasta internacional. La tarea consiste entonces en reconocer los elementos que han fortalecido esta integración y fomentarla.

Se identifican mínimos elementos de articulación productiva en algunas ramas económicas, sin embargo, la interrelación entre los agentes locales productivos, político administrativos y socioculturales es incipiente aún y se requiere mucho trabajo de consensos, acuerdos y conocimiento de lo que acontece en el territorio local para proponer estrategias y respetar las dinámicas de los actores locales.

De acuerdo a lo encontrado podemos hablar de un excepcional ejemplo de lo que ocurre con los productores de talavera quienes con sus características nos acercan a pensar en la existencia de un modelo propuesto por Marshall y Becattini de Distritos Industriales. Debido a que las actividades productivas 
tradicionales basan su existencia en los valores culturales transmitidos de manera transgeneracional, creando elementos territoriales a partir de los cuales la actividad económica persiste y se adapta para permitir un ingreso a las familias que se desempeñan en dichas actividades.

La recuperación del registro de la denominación de origen de la talavera fue un proceso de gestión que reveló la capacidad de organización de los productores del ramo, así como la evidencia del trabajo coordinado con las autoridades locales y estatales que en su calidad de líderes promovieron la participación de otros actores, como la de investigadores de distintas instituciones de la entidad, para realizar todo el proceso de construcción de pruebas necesarias para concluir con la gestión ante instancias del gobierno federal y con ello recuperar el registro de denominación de origen.

La evidencia anterior despliega la capacidad de autogestión y organización entre los productores de talavera, así como la corresponsabilidad que tienen los distintos niveles de gobierno para promover una forma de organización institucional más participativa. En tal sentido, el liderazgo de los niveles gubernamentales es muy importante para promover una forma de gobierno más participativa, así como la descentralización del poder a los distintos niveles gubernamentales para permitir la gestión y auto-organización de los actores locales.

\section{Conclusiones}

Se requiere formular políticas públicas encaminadas a promover las actividades productivas tradicionales que permitan impulsar su crecimiento y consolidar mercados internos y externos, de tal forma que se generen redes verticales y horizontales.

Así, también es importante atender una de las debilidades que existen en el sector productivo local de la región del sur del estado de Tlaxcala: la de consolidar su posición competitiva en el mercado. Para ello, consideramos que es necesario revertir la escasa posibilidad que tienen los gobiernos locales de acceder a los programas de políticas públicas, federales y estatales, como consecuencia de la centralización. El financiamiento de los programas que incluyen al sector productivo queda concentrado en los gobiernos federal y estatal. Y los recursos que llegan a los gobiernos municipales no van dirigidos a apoyar proyectos productivos que puedan ser construidos y formulados desde las comunidades, de tal forma que la promoción del desarrollo local desde las comunidades y/o municipios queda fragmentada y está destinada a trabajar con escaso presupuesto. Esto conduce a la discrecionalidad de la distribución de los recursos, pues no se pueden sentar bases para hacer una distribución abierta a proyectos de carácter local. Dadas estas condiciones, la gestión de las autoridades locales debe redoblarse. Las autoridades deben generar estrategias 
y detectar organismos que les puedan proveer de recursos financieros para llevar a cabo distintos proyectos productivos. Y deben conocer los programas de carácter federal y estatal que les puedan brindar la posibilidad de promover el desarrollo local, toda vez que la información de los programas federales y estatales que apoyan a proyectos productivos entre los empresarios locales es poco conocida.

Se requieren esfuerzos públicos y privados que logren impulsar a las empresas a fomentar sus estructuras de competencia y profesionalización de servicios. Para ello, la capacitación juega un papel importante. A pesar de ser mínimo el porcentaje de empresas que tienen la posibilidad de acceder a este servicio, mediante el estudio se comprobó que ésta es una ventaja: la capacitación está integrada al proceso de producción y permite la estabilidad al trabajador, además de elevar los niveles de competitividad del territorio.

Una desventaja que encontramos durante la investigación es la centralización en los niveles de administración federal y estatal, de programas sociales, económicos y de capacitación que se convierte en límites al desarrollo local. Esta centralización crea desconocimiento de los programas públicos entre los empresarios locales, autoridades municipales y otros agentes que tendrían la posibilidad de integrarse a proyectos de desarrollo que pudieran involucrar a los microempresarios locales, autoridades locales, organizaciones sociales y educativas.

En tanto no se presente una visión integradora de política pública encaminada a potencializar los recursos locales propuesta y construida con los actores locales y las distintas instancias gubernamentales estamos destinados a continuar enfrentando políticas que bajo el principio de desarrollo continúen fomentado inequidades.

\section{BiBLIOGRAFÍA}

ALBURQUERQUE, F. (2004): "Los proyectos de integración productiva en el proceso de desarrollo territorial. Las experiencias y prácticas en la región", en Taller de la Red de proyectos de fomento a la integración productiva entre PyMES en América Latina y el Caribe. Editado por Banco Interamericano de Desarrollo. Fondo Multilateral de Inversiones. Cartagena de Indias, $\mathrm{n}^{\circ} 6-7$, pp. 2-10.

ALBURQUERQUE, F. (1997): Metodología para el desarrollo económico local. en CEPAL/ILPES. Santiago de Chile, pp. 313-325.

AROCENA, J. (1995): El desarrollo local. Un desafío contemporáneo. Ed. Centro Latinoamericano de Economía Humana (CLAEH), Universidad Católica del Uruguay, Editorial Nueva Sociedad. Caracas, Venezuela. p. 175. IZUZQUIZA, I. (1990): "Introducción: la urgencia de una nueva lógica", en LUHMANN, N. Sociedad y sistema: la ambición de la teoría. Ed. Paidós/ I.C.E.-U.A.B. Barcelona, España. pp. 8-39. 
INSTITUTO NACIONAL DE ESTADÍSTICA GEOGRAFÍA E INFORMÁTICA (2011): Censo de población y vivienda, 2010. México, D.F. http://www. inegi.org. $\mathrm{mx} / \mathrm{sistemas} / \mathrm{mexicocifras} /$ default.aspx? $\mathrm{src}=487 \& \mathrm{e}=29$. [consulta 28/04/2012]

LUHMANN, N. (2007): "El sistema como diferencia", en LUHMANN, N. Introducción a la teoría de sistemas, México Ed. Universidad Iberoamericana/Colección teoría social. pp. 77-126.

LUHMANN, N. (1990): Sociedad y sistema: la ambición de la teoría. Ed. Paidós/I.C.E.-U.A.B. Barcelona, España. p. 16.

MEJÍA, J. (2008): "Epistemología de la investigación social en América Latina". Desarrollos en el siglo XXI". Cinta Moebio, $n^{\circ}$ 31, pp. 1-13.

MONCAYO, E. (2001): "Modelos de desarrollo regional: teorías y factores". Boletín Sociedad Geográfica de Colombia. Academia colombiana de ciencias geográficas. Vol. 45, fasc. 133 pp. 91-116.

PADILLAS, Y. et al: Apreciaciones y evolución del desarrollo local. http:// www.monografias.com/trabajos39/concepto-desarrollo-local/concepto-desarrollo-local2.shtml [consultado 03/05/2006]

ROSALES, R. (2003): “Tlaxcala ¿Un distrito industrial?” Sociología, $n^{\circ} 51$. pp. 131-163.

VÁZQUEZ, A. (2005): Las nuevas fuerzas del desarrollo. Antonio Bosch Editor. Universidad Autónoma de Madrid. Barcelona, España. p. 177.

FRIEDMAN, J. y ROH, W. (1979) en VÁZQUEZ, A. (2005). Las nuevas fuerzas del desarrollo, Antonio Bosch Editor, Universidad Autónoma de Madrid. Barcelona, España, pp. 143-154.

WALLERSTEIN, I. (2002): Conocer el mundo, saber el mundo. El fin de lo aprendido. Una ciencia social para el siglo XXI. Editorial siglo XXI editores. México, D.F. 306 p. 